\title{
Bei Nachholimpfungen dürfen Sie jetzt ganz großzügig sein
}

\author{
Die neuen Empfehlungen der Ständigen Impfkommission (STIKO) enthalten \\ zum ersten Mal Empfehlungen zu Nachholimpfungen bei unbekanntem oder \\ unvollständigem Impfschutz. Dies sollten vor allem die Hausarztpraxen zu \\ einer verstärkten Überprüfung des Impfstatus der Patienten veranlassen. Der \\ Nutzen liegt auf beiden Seiten: Der Impfschutz des einzelnen Patienten und \\ der Bevölkerung insgesamt wird deutlich verbessert, den Arztpraxen winkt ein \\ zusätzliches Honorar außerhalb der Regelleistungsvolumina und ein zusätz- \\ licher Imagegewinn bei den Patienten!
}

- Ein wesentlicher Aspekt der neuen STIKO-Empfehlungen ist, dass die STIKO feststellt: Bei fehlender Dokumentation sollte im Praxisalltag im Interesse der zu schützenden Person davon ausgegangen werden, dass der Impfschutz fehlt. Anamnestische Angaben zu bisherigen Impfungen und durchgemachten Krankheiten (z. B. Masern, Mumps, Röteln) sollen in der Regel als unzuverlässig eingestuft werden. Eine Ausnahme davon sind offensichtlich die Angaben zu Windpocken.

In der Regel sind vor 1970 geborene Erwachsene entweder gar nicht oder nur einmal gegen Masern geimpft. Diese Gruppe soll einmal mit einem Kombinationsimpfstoff Masern/Mumps/Röteln komplettiert werden. Da es sich hier um einen Lebendimpfstoff handelt, gilt es, die Gruppe der HIV-Infizierten zu beachten. Erst ab einer CD4-Zellzahl von über 200/Mikroliter ist eine Impfung möglich.

Ist die Impfung kontraindiziert, ist es umso wichtiger, die Umgebung von Patienten mit schweren Immundefekten umfassend zu impfen. Nicht nur Partner und Familienmitglieder, sondern auch Pflege- und sonstige Personen im Haushalt sollten geimpft werden.

Da von zusätzlichen Impfungen in Zweifelsfällen in der Regel kein erhöhtes Risiko ausgeht, sollen Nachholimpfungen großzü- gig durchgeführt werden. „Überimpfen“ ist ungefährlich und im Vergleich zum Erkrankungsrisiko zu vernachlässigen.

Unter diesem Gesichtspunkt machen "Serologische Titerbestimmungen" nur in Ausnahmefällen wie bei Patienten mit Immundefizienz oder zum Nachweis des HepatitisB-Schutzes Sinn.

\section{Mumpsimpfung: berufliche Indikation ausgeweitet}

Die beruflichen Indikationen zur Mumpsimpfung wurden von der STIKO erheblich ausgeweitet auf praktisch alle Personen, die in Gesundheitsberufen mit der Versorgung von Patienten, in Gemeinschafts- oder Schulungseinrichtungen mit Kindern und jungen Erwachsenen tätig sind.

Diese Empfehlungen gelten insbesondere auch für Arztpraxen und ihr Personal, die mit der Versorgung von Immunsupprimierten betraut sind. Die anvertrauten Patienten sollten durch uns nicht gefährdet werden! Im Verletzungsfall wird die alleinige Auffrischung gegen Tetanus als obsolet betrachtet. Nur sinnvolle Kombinationsimpfstoffe sollten zum Einsatz kommen, damit ein umfassender Impfschutz gewährleistet wird. Die Mindestkombination sollte Tetanus/ Diphtherie sein, häufiger wird die Kombination mit Polio ebenfalls sinnvoll sein. Insbesondere bei älteren Patienten ist die zusätz- liche Kombination mit Pertussis angezeigt. Bei der Impfung gegen MeningokokkenMeningitis hat die STIKO die Erweiterung der Zulassung dieser Impfstoffe auf Kinder ab einem Jahr berücksichtigt. Bisher waren die 4-valenten Konjugatimpfstoffe erst ab dem 11. Lebensjahr zugelassen. Die routinemäßige Impfung aller Kinder im zweiten Lebensjahr mit einem Konjugatimpfstoff gegen Meningokokken C bleibt unverändert bestehen.

Die Empfehlung zur Impfung wurde von der STIKO auf Personengruppen mit erhöhtem Risiko für schwere Meningokokken-Erkrankungen ausgeweitet. Darunter zählen angeborene und erworbene B- und T-Zelldefekte. Aber auch für Personen mit Zustand nach Splenektomie und Reisende in Länder mit hohem Infektionsrisiko oder für Teilnehmer einer Pilgerreise nach Mekka wird die Impfung jetzt dringend empfohlen.

Für die Praxis gilt, dass die Empfehlungen der STIKO für die Impfungen, die nicht zu den Reiseimpfungen zählen oder in die Vorsorgepflicht des Arbeitgebers bei beruflicher Exposition fallen, für die Krankenkassen zur Pflichtleistung werden, da sich der Gemeinsame Bundesausschuss diesen Empfehlungen ohne Wenn und Aber anschließt. Um den Umgang mit den neuen Empfehlungen zu erleichtern, hat die STIKO im Internet ein umfassendes Informationsangebot zur Verfügung gestellt: Neben dem aktuellen Impfkalender finden sich dort zum Download auch Einwilligungserklärungen in die Masern-Mumps-Rötelnund Varizellen-Impfung in 15 Sprachen: www.rki.de/impfen.

HeLmut WaLberT *

- Zum Thema Reiseimpfungen: siehe CME-Beitrag ab S. 66. 